\title{
3D Measurement and Reconstruction based on Structured Light
}

\author{
YU Yongyan ${ }^{1, a}$, SHU Yuqin ${ }^{2, b}$ \\ ${ }^{1}$ Department of Computer Engineering,Huaiyin Institute of Technology,Huaian 223003,China \\ ${ }^{2}$ Huaian Qinyin Decoration company, Huaian 223001,China \\ a Shanshan_yyy@163.com , b 13378402263@qq.com
}

Keywords: Structured Light, Continuous Coding, Phase Shifting, 3D Reconstruction

\begin{abstract}
D measurement is one of the most important topic in computer vision,which be used to recover object surface. Using a calibrated projector-camera pair, a light pattern is projected onto the scene and imaged by the cameras. Since the pattern is coded,correspondences between image points and points of the projected pattern can be easily found.These correspondences are used to triangulate and extract 3D information of the surface. Dense reconstruction can be obtained when combining traditional binary codes with phase shifting techniques, where the same pattern is projected and shifted several times. From phase deviation is possible to extract height of the object, given the distance to a reference plane and calibration parameters.
\end{abstract}

\section{Introduction}

3D measurement is one of the most important topic in computer vision, having a variety of applications such as range sensoring, industrial inspection, reverse engineering, object recognition, 3D map building and etc..The developed solutions are traditionally categorized into contact and non contact. Contact measurement techniques have been used for a long time in reverse engineering and industrial inspections, in which main problems are its slow performance and high cost price ${ }^{1}$.Non contact techniques were developed to cope with this problem,in which techniques can be both active and passive. In passive approaches, the scene is first imaged from two or more points of view and correspondences between the images are found. It is important to mention that the cameras have to be previously calibrated ${ }^{2}$.The main difficulty of this approach is to find correspondences when reconstructing textureless surfaces densely and accurately.But, stereovision is rather limited to make 3D surface maps, due to the problem of finding correspondences.

Fortunately,methods based on structured light came to cope with this problem, creating correspondences and giving specific codewords to every unitary position of the image.In active techniques one of the cameras is substituted by an active device, which projects a structured light pattern onto the scene. This active device is modeled as an inverse camera, so the calibration step is pretty similar to the procedure used in classical stereo vision system ${ }^{3}$. The projected pattern imposes the illusion of texture onto an object, as well as increases the number of points of correspondence from two different perspectives ${ }^{4}$. As the structure of the projected pattern is known, the object can be 3D reconstructed by using one single image, looking for differences between the projected and the recorded pattern. There are different approaches of structured light. The one adopted here below classify the different methods regarding its discrete or continuous strategy followed to create the pattern, despite a posterior subdivision in temporal, spatial or frequency based codification is followed.

During the last years, some of the continuous coding techniques have been profusely developed. Therefore,our aim in this paper is to measurement 3D object via continuous coding techniques based on Structured Light.

\section{Continuous Coding Methods}


Coded structured light systems are based on the projection of one pattern or sequence of pattern that univecatelly determine the deviation between projected and received pixel positions.This is provided by a phase deviation value or by a codeword specific for each pixel.Every coded pixel has its own codeword within the no repetitivity region, so there is a direct mapping from the codewords to the corresponding coordinates of the pixel in the pattern.Pattern projection techniques differ in the way in which every point in the pattern is coded and decoded; that is, the spatial, temporal or frequencial strategy followed to create the codeword,and whether they use one axis or two axis codification, color coding or other characteristics.

In continuous coding every pixel has its own value within a region,so the three techniques can provide dense reconstruction under certain constraints. The size of this region is imposed by the periodicity of the patterns employed or can be the whole image.

There are two main groups within this continuous coding:those using periodic patterns where the codeword is taken from the phase deviation to original projected pattern, and those holding the information of a pixel in its intensity or color value.First group are conceived to work with smooth surface, as the pattern presents periodicity in its design. Second group have absolute codification instead. Within phase based methods, there exist two different groups depending of the techinque used to decode: phase shifting approaches5 and frequency transform methods6.

Continuous coding strategies use grading and usually periodic gray-code sequences.The 3D depth of a given point in the image is determined by its gray value and the deviation of this value with respect to the projected pattern.Grading methods, which belongs to spatial coding methods, project one single pattern where the codeword is hold only in every projected point, for that pixel.They are useful for achieving high spatial resolution and few projecting patterns. However,error sensitivity is extremely high in these methods, as the received pattern can be affected by many factors, like resolution of the projector or sensor device or reflectivity of the surface.Some authors use non-standard optical devices to increase the resolution 7.

Given a projected fringes pattern, an analysis of fringes deformation is performed in order to determine phase deviation. Having this, 3D structure of the object can be recovered.It is important to note that these methods assume smooth surfaces, because high slopes in the structure would cause errors in the recovering step8. Due to this fact patterns show periodicity in its conception. Furthermore, these methods show good resistance to ambient light and resistance to reflection variation, due to the grayscale nature of projected patterns.However, these techniques present the problem of having to project at least three patterns to suppress the effect of albedo and to recover the phase deviation.

\section{Phase Shifting Methods}

When projecting a sinusoidal grating onto an object or a surface, every point along a line normal to the grating point can be characterized by a unique phase value regarding the projected pattern. Any non flat 3D shape will cause a deformation in the recorded pattern with respect to the projected one, which is recorded as a phase deviation. This phase deviation provides us information about the illuminated shape. Performing an accurate measure of this phase using phase modulation methods and determining points on the reference plane and the object having identical phases, object shape can be recovered. In spatial decoding techniques the pattern has to be shifted and projected several times, in order to extract phase deviation. Compared to other structured light techniques, they present goodnesses like resistance to ambient light and resistance to reflection variation, due to the grayscale nature of projected patterns. There are two groups of phase shifting techqniues, depending of the frecuencial nature of projected patterns: simple phase shifting methods and multiple phase shifting methods. In the later, more than one frequency are used to create the patterns, thus giving spetial characteristics in the phase extraction procedure.

\subsection{Single Phase Shift Methods}

These techniques use only one frequency to create the sequence of patterns. In order to recover phase deviation, the pattern is projected several times, each one shifted from the previous by a 
factor of $2 \pi / N$, being $N$ the total number of projections.

$$
I^{p}\left(x^{p}, y^{p}\right)=A^{p}+B^{p} \cos \left(2 \pi f_{\phi} y^{p}-2 \pi n / N\right)
$$

where $A^{p}$ and $B^{p}$ are the projection constants and $\left(x^{p}, y^{p}\right)$ the projection coordinates.

The received intensity images from the object surface after projections are

$$
I(x, y)=\alpha(x, y)\left[A+B \cos \left(2 \pi f_{\phi} y^{p}+\phi(x, y)-2 \pi n / N\right)\right]
$$

Logically, it suffers of intensity and phase deviation, thus being necessary to cancel the effect of different albedo $\alpha$ to correctly extract the phase.

$$
\phi(x, y)=\arctan \left[\frac{\sum_{n=1}^{N} I_{n}(x, y) \sin (2 \pi n / N)}{\sum_{n=1}^{N} I_{n}(x, y) \cos (2 \pi n / N)}\right]
$$

Theoretically, From a minimum of three projected shifted patterns is possible to reconstruct the phase deviation caused by the object shape9.However, it must be noticed that arctangent function returns values between the range $(-\pi, \pi]$, so a phase unwrapping procedure is necessary to work with a non ambiguous phase value out of the wrapped phase.This is the reason why they provide effective dense reconstruction only under the restriction of smoothed surface.It must be noticed the importance of phase unwrapping step, which becomes necessary in these methods.

\subsection{Multiple Phase Shift Methods}

Using more than one frequency in phase shifting methods comes to cope with the uncertainty created in the extracted wrapped phase.Traditionally,three solutions have been adopted: temporal phase unwrapping, hierarchical approach and number-theoretical approaches. The first two create several relative phase maps, while the third one is based on the properties of relative primer numbers and divisibility of integers to create an absolute phase map.

Thus,absolute phase map can be computed from two $\square$ ifferent relative phase maps, where the frequencies employed are relative primitive numbers:

$$
\Phi_{A B S_{1}}=k_{1} C \operatorname{dot} \lambda_{1}+\phi_{R_{1}}=k_{1} C \operatorname{dot} \lambda_{1}+\phi_{R_{1}, I N T}+\phi_{R_{1}, D E C I M A L}=\Phi_{A B S_{1}, I N T}+\phi_{R_{1}, D E C I M A L}
$$

Where $\Phi_{A B S_{1}, I N T}=k_{1} C \operatorname{dot} \lambda_{1}+\phi_{R_{1}, I N T}=\sum_{i=1}^{N} \phi_{R_{i}, I N T} e_{i} \bmod \left(\lambda_{1}, \lambda_{2}\right)$.

From the relative phase maps, absolute phase map is obtained using Eq.(4). Absolute phase map gives a correspondences map that can be compared with the ideal phase shift map. From this, correspondences are found and triangulation is performed. The algorithm was tested for different pairs of frequencies over a flat surface. Also, reconstruction of a footprint and a face were pursued, giving low errors in the $3 \mathrm{D}$ reconstruction ${ }^{10}$.

\section{Experimentation and Results}

The use of structured light for 3D reconstruction has a large number of approaches. There exist a variety of patterns and decoding algorithms. However, they share a set of common characteristics or required steps that every algorithm must follow, and can be categorized in: camera projector calibration,pattern generation, projection and recovery, finding correspondences, triangulation, and surface creation.

Thus, a low-cost structured light system was employed. It is composed of an LCD video projector working at $1024 \times 768$ pixels, a camera and a frame grabber digitizing images at $768 \times 576$ pixels and $3 \times 8$ bits per pixel. A standard PC was used for implementing the algorithms.Continuous coding can achieve dense reconstruction from a set of projected patterns.

Within phase shifting methods, sinusoidal patterns can obtain dense reconstruction due to its continuous nature. However, as results of their intrinsic periodicity they are not able to reconstruct sharp objects containing big slopes. This problem is overcome in multiple phase shifting, where they make use of the remainder Theorem to project patterns of two different frequencies, having a frequency relation. 
Hereby, some qualitative results are presented. The reconstructed object is a statue of a white horse of dimensions $21 \times 15 \times 10 \mathrm{~cm}$. Results are presented in both $3 \mathrm{D}$ cloud of points and surfaces.It must be mentioned that no smoothing step has been applied to the $3 \mathrm{D}$ points or to the surface,in order to compare the results without postprocessing.

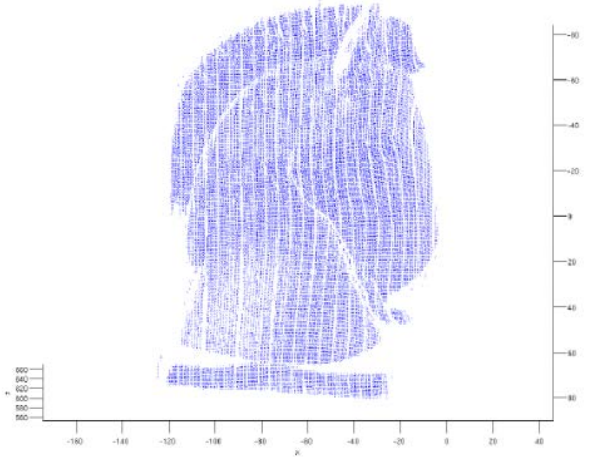

(a) 3D cloud of points

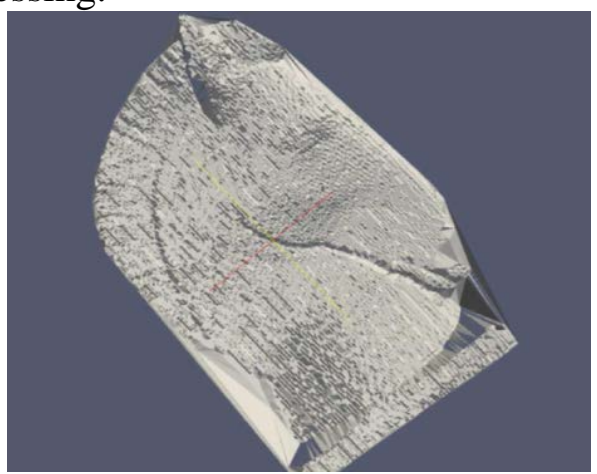

(b)Surface reconstruction

Figure 1: 3D Object reconstruction

The algorithm performs well in presence of slopes, as can be observed in the ears once the image is captured. The holes in the surface correspond to discarded noisy points.

Quantitative results also be analysed reconstructing a white plain at a distance of about $60 \mathrm{~cm}$ to the camera. SVD decomposition was applied to obtain the equation of the 3D plain for every technique and for every reconstruction.

\section{Conclusions}

Non contact measuring using structured light constitutes an alternative to contact methods and laser based techniques, usually more costly and intrusive.Continuous coding permits to achieve dense reconstructions, at the expense of higher number of projected patterns, smoothed surfaces constraint or high sensitivity to noise.Dense reconstruction can be obtained when combining traditional binary codes with phase shifting techniques, where the same pattern is projected and shifted several times. From phase deviation is possible to extract height of the object, given the distance to a reference plane and calibration parameters.Continuous coding strategies dealing with moving objects use frequency multiplexing techniques, or grading.Multiple Phase Shifting approaches are the best solution to have dense reconstruction and absence of periodicity, which could lead to some problems in presence of slopes.

\section{Reference}

${ }^{1}$ J. Vanherzeele, P. Guillaume, and S. Vanlanduit. Fourier fringe processing using a regressive Fourier-transform technique. Optics and Lasers in Engineering, 43(6):645-658,2005.

2 Pages J. Salvi J. and Batlle J. Pattern codification strategies in structured light systems.Pattern Recognition, 37(4):827-849, 2004.

3 S. Zhang and P.S. Huang. Novel method for structured light system calibration. Optical Engineering, 45:083601, 2006.

4 J. Salvi, J. Batlle, and E. Mouaddib. A robust-coded pattern projection for dynamic 3D scene measurement. Pattern Recognition Letters, 19(11):1055-1065, 1998.

${ }^{5}$ Joaquim Salvi Tomislav Pribanic, Sasa Mrvos. Efficient multiple phase shifting pattern for 3D dense acquisition in structured light scanning. 2008.

${ }^{6}$ H.M. Yue, X.Y. Su, and Y.Z. Liu. Fourier transform profilometry based on composite structured light pattern. Optics and Laser Technology, 39(6):1170-1175, 2007.

7 G.S. Spagnolo, G. Guattari, C. Sapia, D. Ambrosini, D. Paoletti, and G. Accardo. Contouring of artwork surface by fringe projection and FFT analysis. Optics and Lasers in Engineering, 33(2):141-156, 2000. 
${ }^{8}$ J. Sun, G. Zhang, Z. Wei, and F. Zhou. Large 3D free surface measurement using a mobile coded light-based stereo vision system. Sensors \& Actuators: A. Physical, 132(2):460-471,2006.

${ }^{9}$ L.S. Wu and Q. Peng. Research and development of fringe projection-based methods in 3D shape reconstruction. Journal of Zhejiang University-Science A, 7(6):1026-1036, 2006.

${ }^{10}$ M.A. Tehrani, A. Saghaeian, and O.R. Mohajerani. A New Approach to 3D Modeling Using Structured Light Pattern. In Information and Communication Technologies: From Theory to Applications, 2008. ICTTA 2008. 3rd International Conference on, pages 1-5,2008. 